
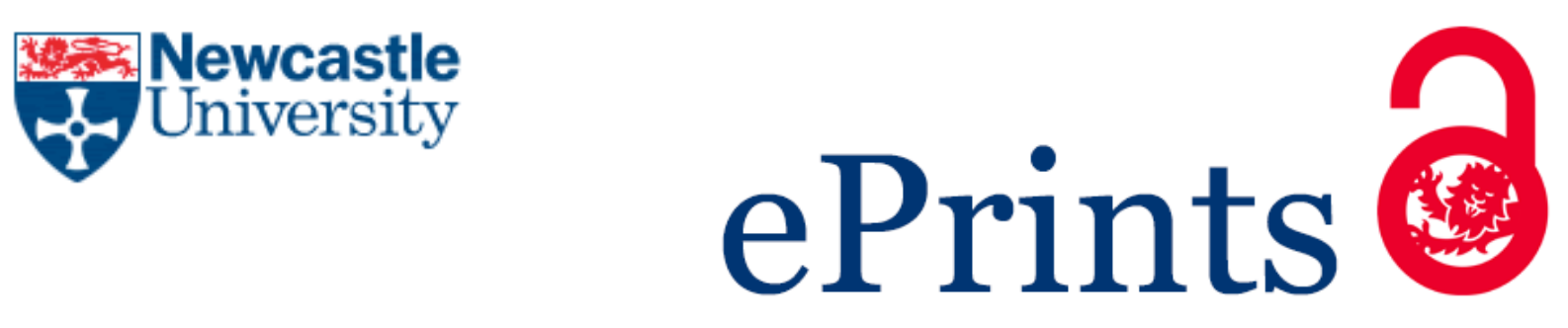

\title{
Penfold TJ.
}

Accelerating Direct Quantum Dynamics using Graphical Processing Units. Physical Chemistry Chemical Physics 2017

DOI: https://doi.org/10.1039/C7CP01473B

\section{Copyright:}

This is the authors' accepted manuscript of an article that was published in its final definitive form by Royal Society of Chemistry, 2017.

DOI link to article:

https://doi.org/10.1039/C7CP01473B

Date deposited:

$31 / 03 / 2017$

Embargo release date:

31 March 2018

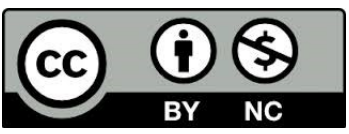

This work is licensed under a Creative Commons Attribution-NonCommercial 3.0 Unported License 

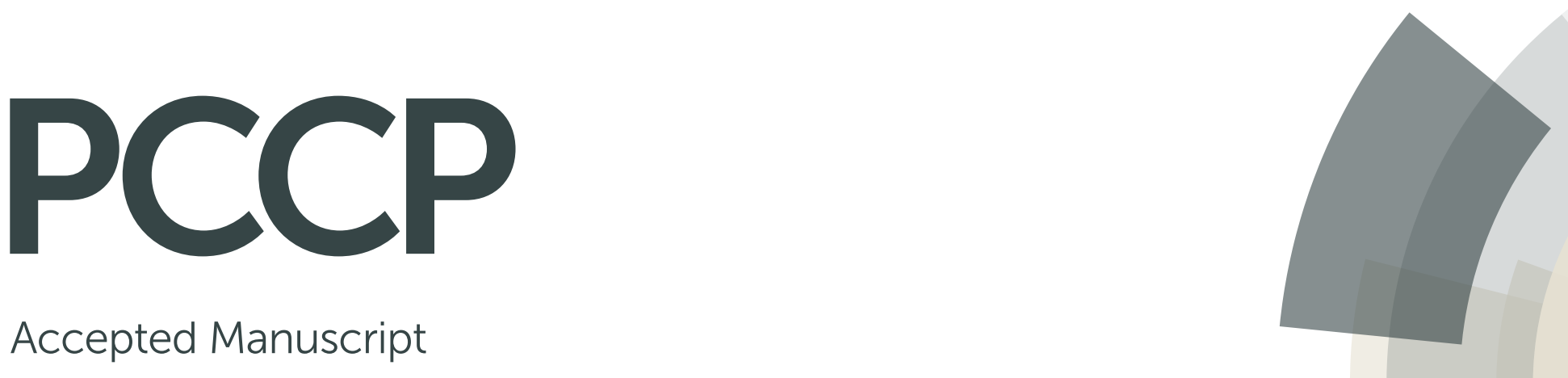

Accepted Manuscript

This article can be cited before page numbers have been issued, to do this please use: T. J. Penfold, Phys. Chem. Chem. Phys., 2017, DOI: 10.1039/C7CP01473B.

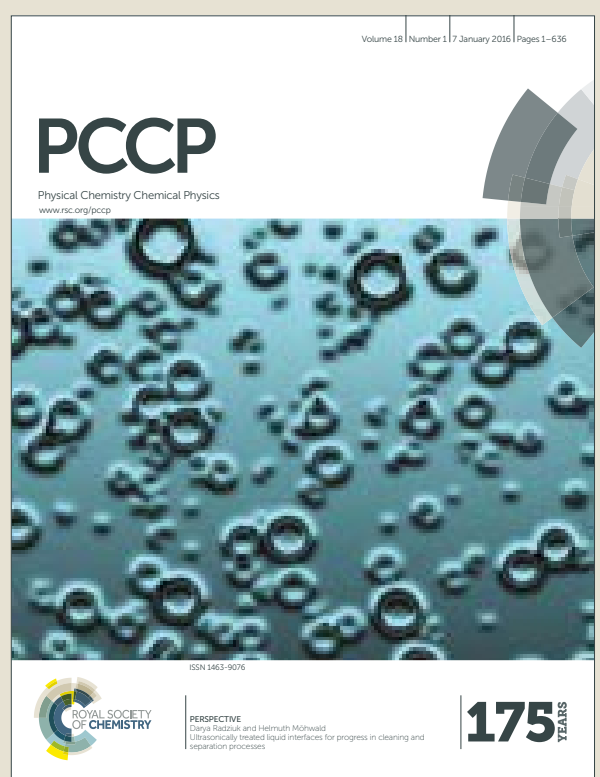

This is an Accepted Manuscript, which has been through the Royal Society of Chemistry peer review process and has been accepted for publication.

Accepted Manuscripts are published online shortly after acceptance, before technical editing, formatting and proof reading. Using this free service, authors can make their results available to the community, in citable form, before we publish the edited article. We will replace this Accepted Manuscript with the edited and formatted Advance Article as soon as it is available.

You can find more information about Accepted Manuscripts in the author guidelines.

Please note that technical editing may introduce minor changes to the text and/or graphics, which may alter content. The journal's standard Terms \& Conditions and the ethical guidelines, outlined in our author and reviewer resource centre, still apply. In no event shall the Royal Society of Chemistry be held responsible for any errors or omissions in this Accepted Manuscript or any consequences arising from the use of any information it contains. 


\title{
Journal Name
}

\section{ARTICLE TYPE}

Cite this: DOI: $10.1039 / x x x x x x x x x x$

\section{Accelerating Direct Quantum Dynamics using Graphi- cal Processing Units}

\author{
T.J. Penfold ${ }^{* a}$
}

Received Date

Accepted Date

DOI: 10.1039/xxxxxxxxxx

www.rsc.org/journalname

\begin{abstract}
Methods using a swarm of Gaussian basis functions to represent the nuclear wavefunction are a very appealing way to solve the time-dependent Schrödinger equation (TDSE) as they avoid the conventional scaling bottleneck of grid-based methods and provide a grid-free trajectory representation of the dynamics understudy. When coupled with direct (on-the-fly) dynamics, these methods offer the ability to simulate quantum dynamics of larger systems in full nuclear configuration space and avoid the requirement of a priori fitting of a potential energy surface. During such simulations, it is often assumed that the limiting factor is the computational cost of the quantum chemistry calculations. To combat this, in the present paper the direct dynamics variational multi-configurational Gaussian (DD-vMCG) method is combined with electronic structure calculations accelerated by Graphical Processing Units (GPUs). For the systems studied, a protonated ammonia dimer and the imidazole dimer, it is shown that the cost of the term responsible for the quantum behaviour of the nuclear dynamics means that the computational time associated with the quantum chemistry quickly becomes a small part of the overall computational time. Using these simulations, an estimated scaling of the VMCG method, with respect to the number of Gaussian basis functions is reported. This can be used to identify when quantum chemistry is the limiting factor and when GPU acceleration will have a significant effect for both ground and excited state simulations.
\end{abstract}

\section{Introduction}

Molecular dynamics is an important tool for modern computational chemistry and is able to calculate a wide variety of observables, such as rates of reaction, which may be compared to experimental data. However, independent of the accuracy for which the electronic degrees of freedom are treated (classical or $a b$ initio), all of these approaches neglect the quantum nature of the nuclei and therefore effects such as zero-point energy and tunnelling cannot be captured. In electronically excited states, the framework of Newtonian nuclear dynamics cannot address nonadiabatic effects arising from the breakdown of the BornOppenheimer approximation ${ }^{1}$.

The inclusion of quantum nuclear effects can be achieved by solving the time-dependent Schrödinger equation. Here the nuclear wavefunction is most commonly represented on a grid of points. However, even with the development of sophisticated algorithms, such as the multi-configurational time-dependent Hartree (MCTDH) approach ${ }^{2}$, these grid-based solutions are limited to a small number of nuclear degrees of freedom owing to the exponential scaling brought about by the direct product ba-

\footnotetext{
${ }^{a}$ School of Chemistry, Newcastle University, Newcastle upon Tyne, NE1 7RU, United Kingdom.tom.penfold@ncl.ac.uk
}

$\mathrm{sis}^{3}$. In addition, the potential must be pre-computed limiting the dynamics to models or very few $(<10)$ degrees of freedom.

To overcome this, many approximate methods based, to a greater or less extent on trajectories have been developed. This removes the requirement to define a grid of points. A notable example is ring-polymer molecular dynamics ${ }^{4}$, which provides a framework for efficiently incorporating quantum nuclear effects on top of classical nuclear motion in condensed-phase systems. This is generally only applicable to a single potential energy surface, however recent work has illustrated it capabilities beyond the Born-Oppenheimer approximations ${ }^{5,6}$. In the excited state, nonadiabatic transitions, which are formally excluded within the framework of Newtonian nuclear dynamics, can be incorporate using approaches such as the popular Tully's Trajectory Surface Hopping (TSH) ${ }^{7,8}$ method, widely adopted due to its efficiency and accuracy in many cases. Here, the nuclear wavepacket is represented by a swarm of classical trajectories, which are able to hop between different excited state surface according to a stochastic algorithm calculated as a function of the nonadiabatic couplings.

An alternative approach is to start from the time-dependent Schrödinger equation (TDSE) and adopt an expansion of the nuclear wavefunction in terms of Gaussian basis functions. These approaches derive from the work of Heller ${ }^{9,10}$ and include the de- 
localised nature of quantum mechanics, while conveniently maintaining the localised trajectory representation of classical mechanics. Recently a number of sophisticated on-the-fly approaches based upon Gaussian basis functions (GBF) have been developed. These include multiple spawning ${ }^{11,12}$, coupled-coherent states (CCS) ${ }^{13}$, multi-configurational Ehrenfest (MCE) ${ }^{14}$ and the multiple cloning method ${ }^{15}$. Crucially, as these methods perform quantum dynamics without the use of direct product grids, they avoid the exponential scaling associated with quantum dynamics. Indeed, the scaling of the multiple spawning approach has been described as approximately quadratic with respect to the number of $\mathrm{GBF}^{12}$.

A unique method among this class of GBF schemes is the direct dynamics variational multi-configurational Gaussian (DD-vMCG) approach. This achieves an accurate description of nuclear quantum dynamics by adopting a variational solution to the TDSE. Indeed, it should be considered as the trajectory based version of the MCTDH method. While conceptually similar to the spawning method, which adopts the same wavefunction ansatz, this approach retains the coupling between these GBF and the evolution of the expansion coefficients. The GBF can therefore be considered as quantum trajectories, in contrast the the multiple spawning, whose basis functions follow classical trajectories. As a result, vMCG is expected to converge quicker than spawning.

The favourable scaling and the absence of any requirements to pre-compute the potential energy surface brought about using direct dynamics methods in principal shifts the burden of computational load of the simulations onto the time required for the quantum chemistry calculations at each time step of the dynamics. This has the possibility to be significantly reduced owing to the recent progress made with exploiting the massively parallel and high memory bandwidth architecture of Graphical Processing Units (GPUs). These characteristics are favourable for application in arithmetic-intensive computations which can be highly parallelised. Consequently GPUs have found a wide range of applications within molecular modelling ${ }^{16-18}$. Indeed, quantum chemistry calculations exploiting the advantages of GPUs can show over 2 orders of magnitude speed-up compared to traditional CPU computations ${ }^{19-21}$.

In this paper the DD-vMCG method is combined with GPUaccelerated quantum chemistry as implemented within the Terachem code ${ }^{22,23}$. The speed-up that can be achieved is illustrated for two model systems, a protonated ammonia dimer and the imidazole dimer. Despite the reduced cost of the quantum chemistry calculations, it is shown that the computational cost of the quantum potential associated with the nuclear dynamics means that the computational time associated with the quantum chemistry quickly becomes a small part of the overall computational time. These results are analysed to derive an approximate scaling of the vMCG approach with respect to the number of Gaussian basis functions which can be used to identify when quantum chemistry is the limiting factor and when GPU acceleration will have a significant effect for both ground and excited state simulations.

\section{Methods and Computational Details}

\subsection{Computational Details}

All simulations have been performed using the DD-vMCG as implemented within a development version of the Quantic quantum dynamics package ${ }^{2,24}$. The details of the vMCG approach in the context of the present work is discussed in section 2.2. The quantum chemistry was performed using Gaussian09 (CPU) ${ }^{25}$ or Terachem (GPU accelerated) ${ }^{21}$. All quantum chemistry was performed using a single core of an Intel Xeon E5 Processor (3.50GHz). GPU acceleration used achieved using a NVIDIA Tesla Kepler K40 containing 2880 CUDA Cores. In all cases, the potential was calculated using DFT(PBE0) ${ }^{26,27}$ and 6-311g* basis set. At each time step the energy and gradient is calculated. To avoid unnecessary time-consuming calculations of the Hessian matrix at each time step, the Hessian Update ${ }^{28}$ scheme implemented in Quantics was used ${ }^{29}$. To assist with issues related to singularities and the C-matrix inversion (discussed in section 2.2) we have used the Tikhonov regularisation approach ${ }^{30,31}$. All simulations were initiated using a chosen nuclear configuration and zero initial momentum in the nuclear wavepacket.

\subsection{Variational Multi-configurational Gaussian (vMCG)}

In this section an overview of the vMCG method is provided. For more details, readers are referred to refs ${ }^{29,32-35}$. The ultimate aim is to solve the time-dependent Schödinger equation:

$$
i \hbar \frac{\partial}{\partial t} \Psi(\mathbf{r}, \mathbf{R}, t)=\hat{\mathscr{H}} \Psi(\mathbf{r}, \mathbf{R}, t)
$$

where $\hat{\mathscr{H}}$ and $\Psi(\mathbf{r}, \mathbf{R}, t)$ are the full molecular Hamiltonian and Wavefunctions, respectively.

The derivation of the vMCG method begins by representing the molecular wavefunction, $\Psi(r, R, t)$, as the product of the electronic wavefunction $\left(\psi^{(s)}(\mathbf{r})\right), \chi_{j}^{(s)}(\mathbf{R}, t)$ is a swarm of $j$ Gaussian basis functions (GBF), weighted by time-dependent coefficients $\left(A_{j}^{(s)}\right)$ :

$$
\Psi(\mathbf{r}, \mathbf{R}, t)=\sum_{s} \sum_{j} A_{j}^{(s)}(t) \psi^{(s)}(\mathbf{r}) \chi_{j}^{(s)}(\mathbf{R}, t)
$$

This ansatz contains, as illustrated by the sum over $s$ electronic states, a set of GBFs for each state. This is the multi-set formalism. As this work only considers dynamics upon a single Born-Oppenheimer surface, by default, the single set formalism is adopted. Consequently, the $s$ superscript is dropped from all future equations and the ansatz becomes:

$$
\Psi(\mathbf{r}, \mathbf{R}, t)=\sum_{j} A_{j}(t) \psi(\mathbf{r}) \chi_{j}(\mathbf{R}, t)
$$

The GBF are expressed:

$\chi_{j}(\mathbf{R}, t)=\prod_{\alpha}\left(2 \pi \sigma_{\alpha}^{2}\right)^{-1 / 4} \exp \left(-\frac{1}{4 \sigma_{\alpha}^{2}}\left[R_{\alpha}-R_{j \alpha}(t)\right]^{2}+i \frac{p_{j \alpha}}{\hbar} R_{\alpha}+i \gamma\right)$

where $\mathbf{R}$ and $\mathbf{p}$ are the position and momentum of the GBFs along each degree of freedom, $\alpha . \sigma$ is the wavepacket width and $\gamma$ is the phase term which carries the quantum information. In the interest of numerical stability, $\sigma$ is kept fixed. The nuclear wave- 
function is therefore a superposition of frozen GBFs ${ }^{10}$.

The vMCG equations of motion (EOM) are derived using this ansatz within the Dirac-Frenkel variational principle ${ }^{36,37}$ :

$$
\begin{gathered}
\left\langle\delta \Psi(t)\left|\hat{\mathscr{H}}-i \frac{\partial}{\partial t}\right| \Psi(t)\right\rangle=0 \\
\langle\delta \Psi(t)|\hat{\mathscr{H}}| \Psi(t)\rangle=i\left\langle\delta \Psi(t) \mid \frac{\partial}{\partial t} \Psi(t)\right\rangle
\end{gathered}
$$

guaranteeing variationally optimal evolution of the nuclear wavefunction. To obtain the EOMs, this must be applied to both the GBFs and expansion coefficients. Firstly in terms of the expansion coefficients, the left hand side of Eq. $5 \mathrm{~b}$ is:

$$
\begin{gathered}
\langle\delta \Psi|\hat{\mathscr{H}}| \Psi\rangle=\sum_{l}\left\langle\chi_{j}|\langle\psi|\hat{\mathscr{H}}| \psi\rangle| \chi_{l}\right\rangle A_{l} \\
=\sum_{l}\left\langle\chi_{j}|\hat{\mathscr{H}}| \chi_{l}\right\rangle A_{l} \\
=\hat{\mathscr{H}}_{j l} A_{l} .
\end{gathered}
$$

Where $\hat{\mathscr{H}}_{j l}$ are the elements of the Hamiltonian matrix. The right hand side is:

$$
\begin{array}{r}
i\left\langle\delta \Psi \mid \frac{\partial \Psi}{\partial t}\right\rangle=i\left\langle\chi_{j}\left|\langle\psi| \sum_{l}\left(\dot{A}_{l}\left|\chi_{l}+A_{l}\right| \dot{\chi}_{l}\right\rangle\right) \mid \psi\right\rangle \\
=i \sum_{l}\left(\left\langle\chi_{j} \mid \chi_{l}\right\rangle \dot{A}_{l}+\left\langle\chi_{j} \mid \dot{\chi}_{l}\right\rangle \dot{A}_{l}\right) \\
=i \sum_{l}\left(S_{j l} \dot{A}_{l}+\tau_{j l} A_{l}\right)
\end{array}
$$

Here $S_{j l}$ are elements of the overlap matrix and $\tau_{j l}$ are elements of the time-derivative matrix. Therefore the EOM for the expansion coefficients is expressed:

$$
\begin{gathered}
i \sum_{l} S_{j l} \dot{A}_{l}=\sum_{l}\left(H_{j l}-i \tau_{j l}\right) A_{l}+H_{j l} A_{l} \\
i \sum_{l} \dot{A}_{l}=S_{j l}^{-1} \sum_{l}\left(H_{j l}-i \tau_{j l}\right) A_{l}+H_{j l} A_{l}
\end{gathered}
$$

It is noted that this differs from the standard MCTDH equations only by the requirement to address, using $S_{j l}^{-1}$, the nonorthogonality of the GBFs. For the GBFs, or more specifically their parameters (e.g. position, momentum, phase) denoted with a composite character $\Lambda$, the same approach is applied. The left hand side of Eq. $5 \mathrm{~b}$ is:

$$
\begin{array}{r}
\langle\delta \Psi|\hat{\mathscr{H}}| \Psi\rangle=\sum_{l} A_{j}^{*} A_{l}\left\langle\frac{\partial \chi_{j}}{\partial \Lambda_{j \alpha}}|\langle\psi|\mathscr{H}| \psi\rangle| \chi_{l}\right\rangle \\
=\sum_{l} \rho_{j l}\left\langle\frac{\partial \chi_{j}}{\partial \Lambda_{j \alpha}}|\mathscr{H}| \chi_{l}\right\rangle \\
=\sum_{l} \rho_{j l} \mathscr{H}_{j l}^{(\alpha 0)}
\end{array}
$$

where $\rho_{j l}$ are elements of the density matrix and $\mathscr{H}_{j l}^{(\alpha 0)}$ are matrix elements of the mean-field Hamiltonian operator. The right hand side of Eq. 5b, in terms of the GBF parameters is:

$$
\begin{aligned}
i\left\langle\partial \Psi \mid \frac{\partial \Psi}{\partial t}\right\rangle= & i \sum_{l} A_{j}^{*} \frac{\partial \chi_{j}}{\partial \Lambda_{j \alpha}} \mid\left\langle\psi\left|\left(\dot{A}_{l}\left|\chi_{l}\right\rangle+A_{l}\left|\dot{\chi}_{l}\right\rangle\right)\right| \psi\right\rangle \\
& \left.=i \sum_{l} A_{j}^{*}\left\langle\frac{\partial \chi_{j}}{\partial \Lambda_{j \alpha}}\right|\left(\dot{A}_{l}\left|\chi_{l}+A_{l}\right| \dot{\chi}_{l}\right\rangle\right) \\
& =i \sum_{l} A_{j}^{*} \dot{A}_{l} S_{j l}^{(\alpha 0)}+i \sum_{l} \rho_{j l}\left(\sum_{\beta} \dot{\Lambda}_{l \beta} S_{j l}^{(\alpha \beta)}\right)
\end{aligned}
$$

Therefore the EOM for the parameters of the GBF is expressed:

$$
i \sum_{l} \rho_{j l}\left(\sum_{\beta} \dot{\Lambda}_{l \beta} S_{j l}^{(\alpha \beta)}\right)=\sum_{l} \rho_{j l} H_{j l}^{\alpha 0}-i \sum_{l} A_{j} \dot{A}_{l} S_{j l}^{\alpha 0}
$$

Substituting $\dot{A}_{l}$ from Equation $8 \mathrm{~b}$, we arrive at:

$$
\begin{array}{r}
i \sum_{l} \rho_{j l}\left(\sum_{\beta} S_{j l}^{\alpha \beta}-\left[S^{(\alpha 0)} \cdot S^{-1} \cdot S^{0 \beta}\right]_{j l}\right) \dot{\Lambda}_{l \beta}= \\
\sum_{l} \rho_{j l}\left(H_{j l}-\left[S^{(\alpha 0)} \cdot S^{-1} \cdot H\right]_{j l}\right)
\end{array}
$$

This is commonly simplified to ${ }^{29}$ :

$$
i \dot{\Lambda}=C^{-1} Y
$$

where

$$
C_{j \alpha, l \beta}=\rho_{j l}\left(\sum_{\beta} S_{j l}^{\alpha \beta}-\left[S^{(\alpha 0)} \cdot S^{-1} \cdot S^{0 \beta}\right]_{j l}\right)
$$

and

$$
Y_{j \alpha}=\sum_{l} \rho_{j l}\left(H_{j l}-\left[S^{(\alpha 0)} \cdot S^{-1} \cdot H\right]_{j l}\right)
$$

In the context of the future discussions within the paper and drawing the connections between the vMCG and other GBF approaches, such as multiple spawning, one can separate the propagation of the Gaussian parameters into uncorrelated (classical) and correlated (quantum) terms ${ }^{34}$. Indeed, besides the timedependence of the basis set size of the multiple spawning algorithm, it is the inclusion of the quantum correction on the nuclear motion which captures all the key differences between vMCG and multiple spawning. This is to say that in the former the GBF are correlated and follow quantum trajectories, while in the latter they are uncorrelated and therefore follows classical motion. This division is achieved using a power series expansion in terms of Gaussian moments leading to:

$$
i \dot{\Lambda}=X_{0}+C^{-1} Y
$$

The first term, $X_{0}$ contains the terms responsible for the separable classical motion of the GBF, while $C^{-1} Y$ contains all of the nonclassical parts of the nuclear dynamics.

\section{Results}

\subsection{Protonated Ammonia Dimer}

In the first example the dynamics of a protonated ammonia dimer, $\left[\mathrm{H}_{3} \mathrm{~N}-\mathrm{H}-\mathrm{NH}_{3}\right]^{+}$is studied. This is performed in unconstrained 
nuclear configuration space and therefore contains 21 nuclear degrees of freedom. A similar study was recently performed by Tavernelli ${ }^{38}$ using a Bohmian dynamics based approach. In this case the extra proton was initially placed between two ammonia molecules separated by a fixed $\mathrm{N}$ to $\mathrm{N}$ distance of $2.63 \AA$. The constraint on the N-N distance was imposed to stabilise the system in a reactive configuration with the aim of enhancing the probability of proton transfer. In the present case the overriding interest is the computational cost of the method and the effect of GPU acceleration. In addition, to ensure a fair comparison between the costs of classical and quantum trajectory motion, it is preferred that the dynamics does not exhibit significant quantum nuclear effects. Consequently, the dynamics, performed for $50 \mathrm{fs}$ were initiated from the PBE0 optimised geometry, with the proton distance slightly compressed (by $0.13 \AA$ ) towards the nitrogen on the closest ammonia monomer to initiate the dynamics.
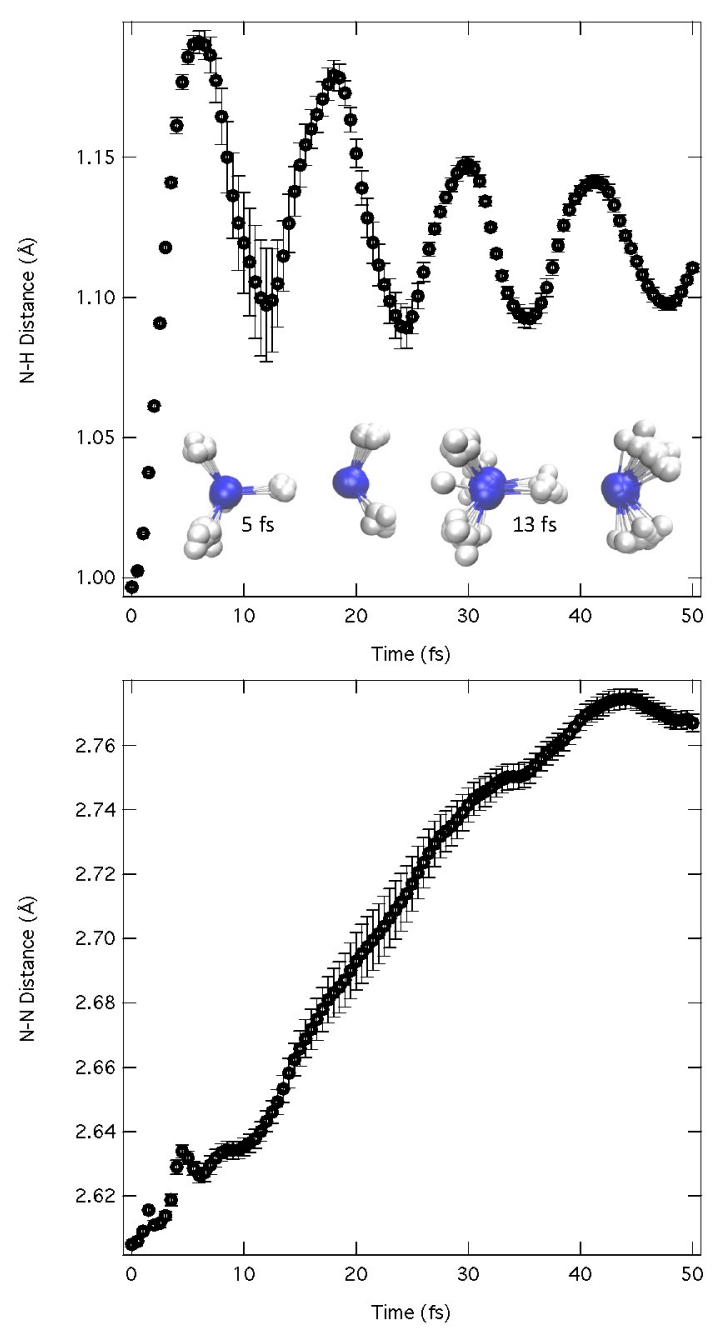

Fig. 1 Upper: The N-H distance of the additional proton during the $50 \mathrm{fs}$ of DD-vMCG simulations with 40 Gaussian basis functions. The error bars represent two times the standard deviation of their average position. Inset are snapshots of the atomic position of each Gaussian basis functions after 5 and 13 fs. Lower: The N-N distance for the same simulation. The error bars also represent two times the standard deviation in their position.
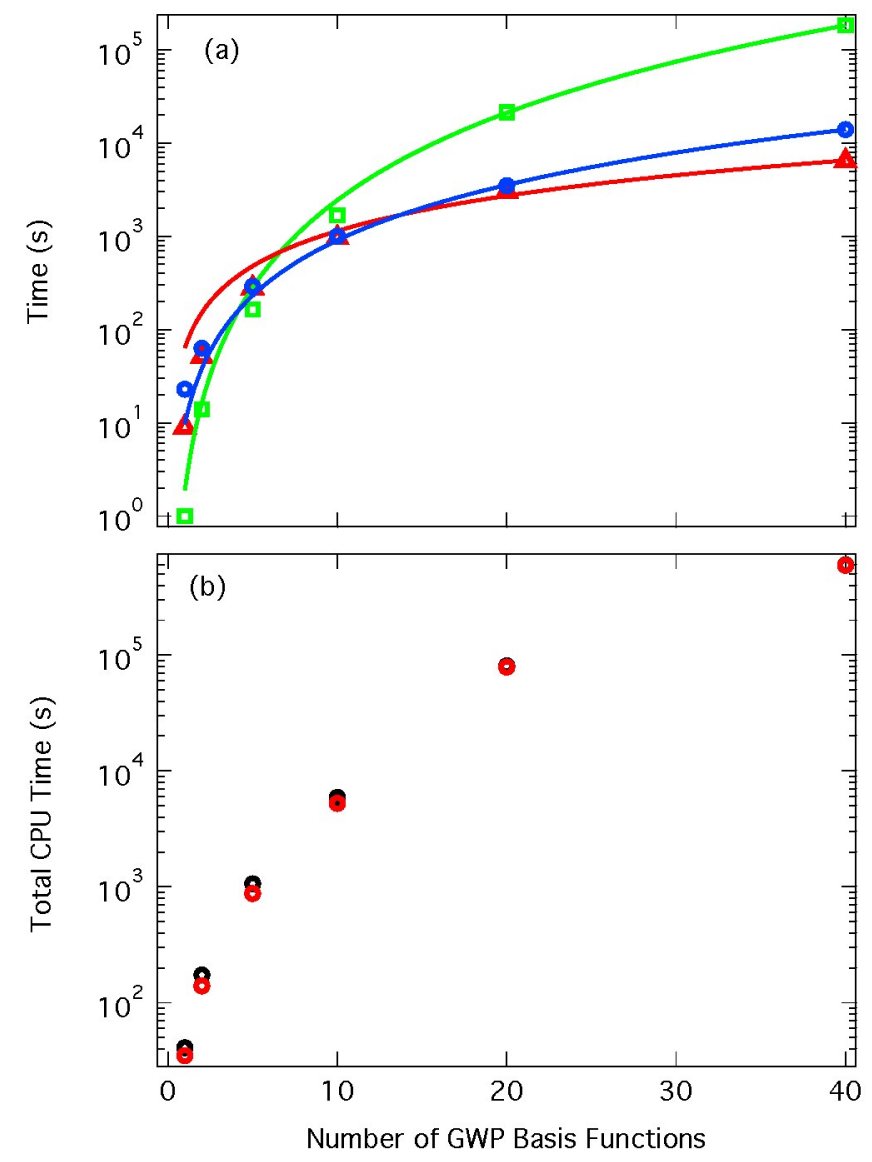

Fig. 2 (a) The time (on a logarithm scale) of each component of the DD-vMCG simulation for the protonated ammonia dimer as a function of the number of Gaussian basis functions. The three components are: the classical Gaussian motion ( $\mathscr{T}_{C}$, blue ), the quantum correction ( $\mathscr{T}_{Q}$, green) and the quantum chemistry $\left(\mathscr{T}_{Q C}^{G P U}\right.$, red). (b) A comparison of the total computational time (on a logarithm scale) as a function of the number of Gaussian basis functions for the GPU accelerated (red) and CPU (black) DD-vMCG simulations.

Figure 1 (upper) shows the $\mathrm{N}-\mathrm{H}$ distance of the additional proton during the first $50 \mathrm{fs}$ of the simulations. Snapshots of the dynamics after 5 and $13 \mathrm{fs}$ are shown inset for the simulation composed of $40 \mathrm{GBF}$. This shows, as expected, large amplitude oscillations which is damped with time as energy is distributed throughout the molecular complex and the $\mathrm{N}-\mathrm{H}$ bond relaxes towards it equilibrium length of $1.13 \AA$. As shown in Figure 1 (lower), this motion is accompanied by a elongation of the N-N bond length during the first $50 \mathrm{fs}$. This separation hinders the proton transfer from one ammonia moiety to the other.

Table 1 shows the computational time associated with each component of the simulations. Despite the small size of the system the reduction in the time for the quantum chemistry calculations $\left(\mathscr{T}_{Q C}\right.$ ) is clearly observed, especially as the number of GBF increases. Indeed for $40 \mathrm{GBF}$ it is close to $50 \%$. However, it is also apparent that the cost associated with the quantum correction to the nuclear dynamics $\left(\mathscr{T}_{Q}\right)$, which as shown in Figure 2 (upper) scales at a much faster rate as these simulations reach convergence at $40 \mathrm{GBF}$. Indeed, by far the most time consuming 


\begin{tabular}{|c|c|c|c|c|c|}
\hline GWP & $\mathscr{T}_{Q C}^{G P U}$ & $\mathscr{T}_{Q C}^{C P U}$ & $\mathscr{T}_{C}$ & $\mathscr{T}_{Q}$ & Speed Up \\
\hline 1 & 9 & 15 & 23 & 3 & 1.17 \\
2 & 51 & 85 & 63 & 26 & 1.26 \\
5 & 279 & 465 & 291 & 307 & 1.21 \\
10 & 963 & 1605 & 1004 & 3300 & 1.12 \\
20 & 3021 & 5035 & 3511 & 72387 & 1.03 \\
40 & 6438 & 10730 & 14010 & 576633 & 1.01 \\
\hline
\end{tabular}

Table 1 Table showing the time (in secs) of each component of the DD-vMCG simulation and the total speed-up provided by the GPU quantum chemistry for the protonated ammonia dimer as a function of the number of Gaussian basis functions. The speed-up factor is the total time for the dynamics using CPU's divide by the total time for the dynamics using GPU's.

part of the simulations is this quantum aspect. Within the time scale of the dynamics this is not associated with issues related to stability of the C-matrix inversion.

Importantly, the effect of this is seen in the overall speed up of the calculations (the speed-up factor is the total time for the dynamics using CPU's divide by the total time for the dynamics using GPU's.The speed-up factor is the total time for the dynamics using CPU's divide by the total time for the dynamics using GPU's). This is reduced for larger number of GBF as the quantum correction becomes more time consuming. This can be seen clearly in Figure 2 (lower) showing the time for the dynamics performed using CPU or GPU quantum chemistry. The differences, admittedly minimised in appearance by the logarithm scale of the $\mathrm{y}$-axis, are very small. This is not unexpected given the size of the system, however it is interesting to note that if the coupling between the GBF are neglected, i.e. the quantum aspect of the GBF motion, then $\mathscr{T}_{Q}$ would not be present and one would observe a reasonably significant speed up ( $\sim 21 \%$ for $40 \mathrm{GBF})$.

\subsection{Imidazole Dimer}

The second example is an isolated dimer of imidazole, recently studied using broadband rotational spectroscopy and high-level quantum chemistry ${ }^{39}$. Imidazole $\left(\mathrm{C}_{3} \mathrm{H}_{4} \mathrm{~N}_{2}\right)$ is a five-membered, aromatic ring that contains both pyrrolic and pyridinic nitrogen atoms. In the present case, the PBE0/6-311g* optimised geometry is adopted and the dynamics are initiated with a proton transferred between the pyrrolic nitrogens on the imidazole units (Figure 3 upper inset). Consequently, this does not represent dynamics one would observe under normal conditions. Instead, for the same reason as the protonated ammonia dimer, it has been chosen so that the quantum and the classical dynamics will be very similar enabling a fair comparison between the cost of the quantum and classical corrections. A one dimensional potential along the proton transfer coordinate is shown in Figure 3 inset and illustrates the barrierless potential leading from the starting structure to the global minimum identified in ref. ${ }^{39}$.

Figure 3 (upper) shows the $\mathrm{N}-\mathrm{H}$ distance (labelled inset) as a function of time. The N-N distance between the two pyrrolic nitrogens is shown in Figure 3 (lower). The points are the distance averaged over the $40 \mathrm{GBF}$ and the error bars are 2 times the standard deviations in the position. There is an immediate increase in

\begin{tabular}{|c|c|c|c|c|c|}
\hline GWP & $\mathscr{T}_{Q C}^{G P U}$ & $\mathscr{T}_{Q C}^{C P U}$ & $\mathscr{T}_{C}$ & $\mathscr{T}_{Q}$ & Speed Up \\
\hline 1 & 161 & 966 & 199 & 3 & 3.22 \\
2 & 382 & 2290 & 580 & 44 & 2.90 \\
5 & 950 & 5698 & 2750 & 904 & 2.03 \\
10 & 1484 & 8904 & 9703 & 8560 & 1.38 \\
20 & 3144 & 18862 & 48516 & 125998 & 1.09 \\
40 & 5116 & 30996 & 182970 & 2135610 & 1.01 \\
\hline
\end{tabular}

Table 2 Table showing the time (in secs) of each component of the DD-vMCG simulation and the total speed-up provided by the GPU quantum chemistry for the imidizole dimer as a function of the number of Gaussian basis functions. The speed-up factor is the total time for the dynamics using CPU's divide by the total time for the dynamics using GPU's.

the $\mathrm{N}-\mathrm{H}$ distance, expected from the potential curve shown inset in Figure 3. This bond oscillates with a period of $10 \mathrm{fs}$, increasingly only slightly from 5-35 fs. This is correlated with an increase in decrease in the N-N distance. At $35 \mathrm{fs}$, another significant increase in the N-H distant is observed and is also accompanied by a faster decrease in the N-N distance. Oscillations with a period of $\sim 10$ fs then begin again around $1.45 \AA$, which given the new shortened N-N distance correspond to a N-H distance of $1 \AA$ with the other pyrrolic nitrogen, consistent with proton transfer with 50 fs. Inset Figure 3 (lower) shows structural snapshots of the dynamics at $10 \mathrm{fs}$ and $30 \mathrm{fs}$. An increase in the spread of the trajectories with time is observed, despite the short time scale of the simulations. Indeed, the motion is dominated by that of the protons and their light nature means motion can be very fast.

Table 2 shows the computational time associated with the simulations as a function of the number of GBF. Here the speed up in the quantum chemistry calculations owing to the use of the GPUs is, as expected, more significant than for the protonated ammonia dimer, with the speed-up being between a factor of $\sim 5-10$. For a small number of GBF, for which the cost associated with the quantum aspect of the nuclear dynamics is small, the overall speed up is significant. This is also highlighted in Figure 4b showing the total computational time of the dynamics performed using a either CPU or GPU architecture. However, as seen for the protonated ammonia dimer, for larger numbers of GBF, the quantum component to the nuclear dynamics $\left(\mathscr{T}_{Q}\right)$ becomes the dominant part of the computational cost. It is also interesting to note that, in contrast to the protonated ammonia dimer, the $\mathscr{T}_{C}$ is more computationally expensive than the quantum chemistry calculations. This is associated with the scaling of this term with the dimensionality of the problem, i.e. imidazole dimer has more degrees of freedom than the protonated ammonia dimer.

\section{Discussion and Conclusions}

In this section we discuss the previous simulations within the wider context of the scaling of vMCG and the potential impact of GPU acceleration. At this point, it is emphasised that the exact scaling of these methods is problem dependent, since the nature and/or complexity of the wave function can be very different along separate degrees of freedom. Consequently, the following discussion acts as a guideline rather than a general rule. It is stressed that the largest uncertainty is the quantum aspect of the 

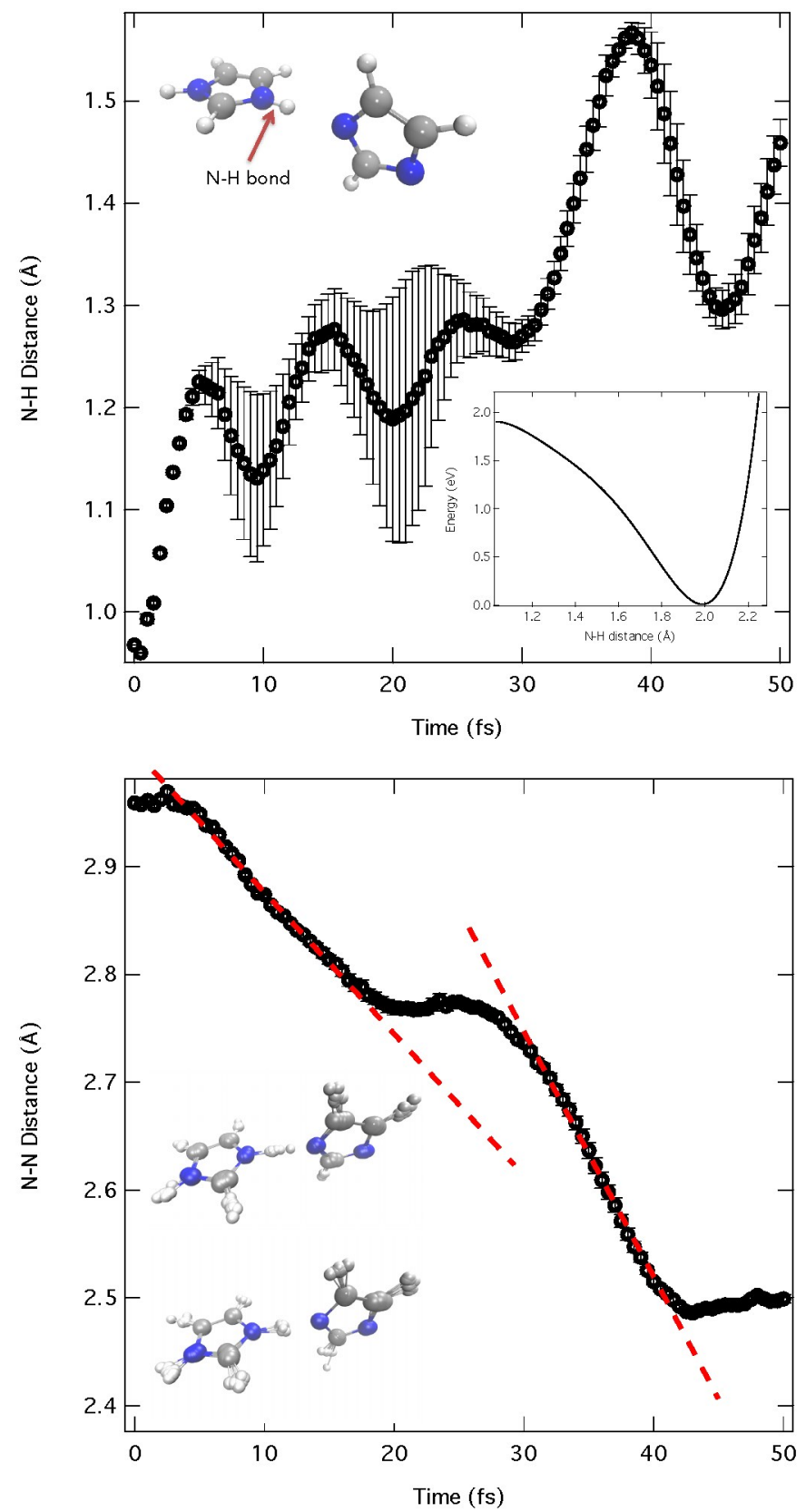

Fig. 3 Upper: The N-H distance (labelled upper inset) during the 50 fs of DD-vMCG simulations with 40 Gaussian basis functions. The error bars represent the standard deviation of their average position. Lower: The N-N distance for the same simulations. The error bars also represent the standard deviation. Inset are snapshots of the nuclear configurations at $10 \mathrm{fs}$ (upper) and $30 \mathrm{fs}$ (lower).

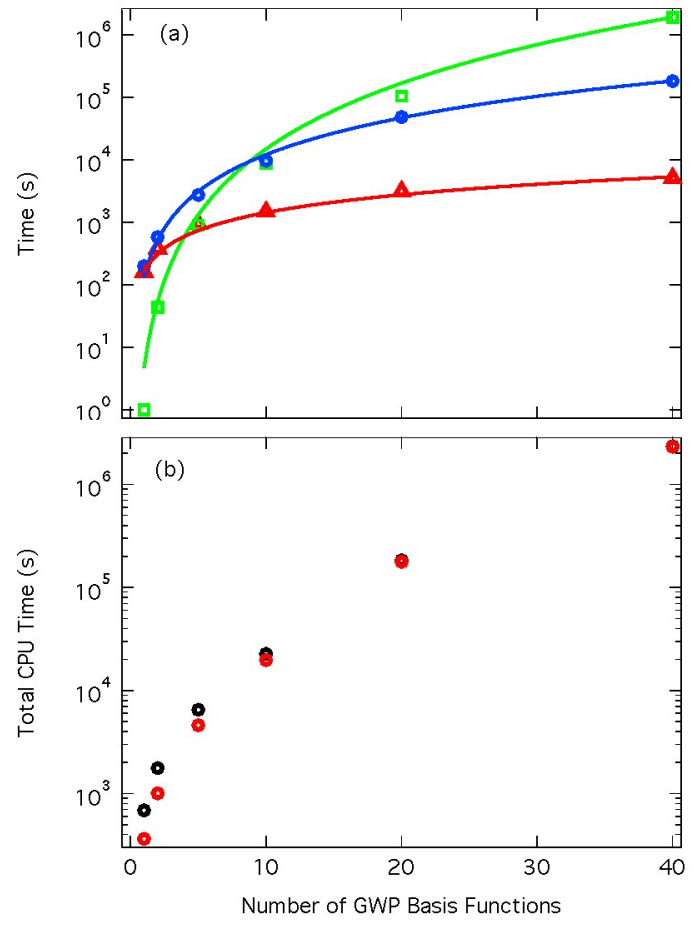

Fig. 4 Upper: The time (on a logarithm scale) of each component of the DD-vMCG simulation for the imidizole dimer dimer as a function of the number of Gaussian basis functions. The three components are: the classical Gaussian motion ( $\mathscr{T}_{C}^{G P U}$, blue ), the quantum correction $\left(\mathscr{T}_{Q}^{G P U}\right.$, green) and the quantum chemistry $\left(\mathscr{T}_{Q C}^{G P U}\right.$, red). Lower: A comparison of the total computational time (on a logarithm scale) as a function of the number of Gaussian basis functions for the GPU accelerated (red) and CPU (black) DD-vMCG simulations.

propagation.

The computational cost of DD-vMCG can be split into 3 parts. The first is the time required for the quantum chemistry ( $\mathscr{T}_{Q C}$ ), the second is the time for the classical (separable) motion of the $\mathrm{GBF}\left(\mathscr{T}_{C}\right)$ and the final is the quantum correction to the nuclear motion $\left(\mathscr{T}_{Q}\right)$. The latter two can be consider to be the first and second terms in Equation 18, respectively. Consequently, the total simulation time can be expressed:

$$
\mathscr{T}=\mathscr{T}_{Q C}+\mathscr{T}_{C}+\mathscr{T}_{Q}
$$

Table 3 shows a power law fit ( $y=A x^{\text {pow }}$ ) of each component $\left(\mathscr{T}_{Q C}^{G P U}, \mathscr{T}_{C}\right.$ and $\mathscr{T}_{Q}$ ) of the simulations as a function of the number of GBF. The times and corresponding fits are shown in Figures 2 and 4 for the protonated ammonia dimer and imidazole

\begin{tabular}{|c|c|c|c|c|c|c|}
\hline Sim & \multicolumn{3}{|c|}{ NH3 dimer } & \multicolumn{3}{c|}{ Imidizole dimer } \\
\hline & $\mathscr{T}_{\mathscr{C}}^{G P U}$ & $\mathscr{T}_{C l}$ & $\mathscr{T}_{Q}$ & $\mathscr{T}_{O C}^{G P U}$ & $\mathscr{T}_{C l}$ & $\mathrm{~T}_{Q}$ \\
\hline A & 63.89 & 9.74 & 1.89 & $\mathbf{1 6 0 . 9 8}$ & $\mathbf{1 3 4 . 2 8}$ & 4.73 \\
pow & 1.25 & 1.97 & 3.11 & 0.95 & 1.96 & 3.48 \\
\hline
\end{tabular}

Table 3 The parameters corresponding to a power law fit $\left(y=A x^{\text {pow }}\right)$ of each component $\left(\mathscr{T}_{Q C}^{G P U}, \mathscr{T}_{C}\right.$ and $\left.\mathscr{T}_{Q}\right)$ of the simulations as a function of the number of GBF. The fits are shown in Figures 2 and 4 for the protonated ammonia dimer and imidazole dimer, respectively. 
dimer, respectively. This reveals that the scaling of the quantum chemistry part of the calculation is approximately linear with the number of Gaussian basis functions:

$$
\mathscr{T}_{Q C} \sim a N_{g w p} \sim n_{q c} t_{q c} N_{g w p}
$$

where the prefactor, $a$, is the product of the time for one quantum chemistry calculation $\left(t_{q c}\right)$ and the number of quantum chemistry calculations $\left(n_{q c}\right)$ required to propagate a single GBF for the dynamics of interest. Here it is important to stress that computational scaling with the absolute size of the system is contained within $t_{q c}$. This will clearly depend on the computational method used. In the context of GPU accelerated quantum dynamics, it is the size of this prefactor which is crucial and is discussed later.

The classical motion of the GBF $\left(\mathscr{T}_{C}\right)$ scales quadratically with $N_{g w p}$ (Table 3):

$$
\mathscr{T}_{C} \sim b N_{g w p}^{2}
$$

This is expected from the previously reported scaling of the Gaussian wavepacket approaches based upon classical motion, such as multiple spawning ${ }^{12}$. Here, the dominating aspect of the computational effort derives from computation of the Hamiltonian matrix and the inversion of the overlap matrix. For a basis set with $\mathrm{N}$ nuclear basis functions, there are $\mathrm{N}^{2}$ Hamiltonian matrix elements, while inversion of the overlap matrix required $\mathscr{O}\left(\mathrm{N}^{3}\right)$ operations. The former is the most time consuming and is the origin of the quadratic scaling. The prefactor $(b)$ also contains a linear dependency with respect to the number of particles in the system $^{12}$.

Finally, the scaling of the quantum part of the Gaussian basis functions (Table 3 ). Here a roughly cubic scaling relationship is observed:

$$
\mathscr{T}_{Q} \sim c N_{g w p}^{3}
$$

for which the source is the inversion of the so called C (not to be confused with c) matrix (Equation 16) ${ }^{29}$. It is noted that a potential way to reduce this produce is by adopting a multi-layer version of the $\mathrm{vMCG}^{40}$ in exactly the same way as MCTDH and its multi-layer variant ${ }^{41}$.

From the previous paragraphs we can approximate that overall, the scaling of DD-vMCG, with respect to the number of GBF's is:

$$
\mathscr{T}=a N_{g w p}+b N_{g w p}^{2}+c N_{g w p}^{3}
$$

It is stressed that each prefactor ( $a, b$ and $c$ ) depends on the dimensionality (size) of the system understudy. The scaling of $a$ with the dimension of the system will also depend on the scaling of the specific quantum chemistry method used. In the context of the present work, the effect of GPU acceleration is manifested in the prefactor, $a$. It is interesting to note that in the case of the imidazole dimer, the time associated with the quantum chemistry calculations could be increased by almost 3 orders of magnitude and this would still only double the total time of the overall simulations. This observation is particularly attractive and relevant in the context of excited state dynamics simulations, for which the quantum chemistry can be considerably more expensive than the DFT used here.

Given the size of $\mathscr{T}_{Q}$ reported in previous sections, it is tempt- ing to state that the effect of GPU acceleration will be more keenly felt in the case of GWP methods based upon classical trajectory evolution, such as multiple spawning, as the significant computational expense of the quantum correction to the nuclear dynamics is removed. Indeed this is compounded by the fact that convergence to the correct dynamics for uncorrelated GBFs is achieved using a larger number of basis functions, which means more quantum chemistry calculations. This has recently been exploited for excited state simulations using GPU accelerated $a b$ initio multiple spawning ${ }^{42,43}$, although no quantitative details on the effect of the speed-up were given. Despite this, for the present implementation of direct dynamics in Quantics, the load on the quantum chemistry calculations is reduced by the use of the database, so that similar geometries are not recalculated, reducing the number of quantum chemistry calculations. Adopting this approach, it was observed that more quantum chemistry calculations were required for vMCG (coupled) rather than uncoupled trajectories. Here, the uncoupled GBF follow classical trajectories and generally exhibit a well order distribution in a small region of classically allowed phase space. In contrast, the vMCG GWP trajectories tend to cover a larger region of phase space. This is the reason for the fast convergence, i.e. the functions move to cover the space as required by the TDSE. But the larger spread also leads to a requirement of more quantum chemistry calculations, making it less clear whether GPU acceleration is more beneficial for GWP methods based upon coupled or uncoupled GBFs.

Finally, the scaling of each component of the DD-vMCG dynamics, shown in Equation 23 illustrates that the effect of GPU accelerated quantum chemistry will be most keenly observed for the smaller numbers of Gaussian basis. In this context, although the wavefunction may not be converged, it has been observed that expectation values, such as position and width of the wavepacket can converge well before the actual wavefunction ${ }^{44-46}$. Therefore a near-quantitive description of the dynamics can emerge within a relatively few number of GBF. Importantly, if they reveal the dominant degrees of freedom active during the dynamics and therefore it will be possible to use such simulations to develop model Hamiltonians using relatively few numbers of GBF. While the dynamics in this case are certainly not converged, they are sufficient to inform the mode selection of models. Recently a few works have proposed approaches that moves in this direction ${ }^{47-49}$.

In conclusion, in this work the DD-vMCG method is combined with electronic structure calculations accelerated by Graphical Processing Units. We show that, as a consequence of the significant number of quantum chemistry calculations required, a significant reduction in the cost of the quantum chemistry components of the calculations is achieved. This is even for the relatively short dynamics of 50 fs studied herein. However, the overall speed-up of the calculations is, especially for larger basis sets is relatively modest. This is associated with the computational cost of the quantum correction to the nuclear dynamics, which scales cubically with the number of GBF, compared to the linear scaling of the number of quantum chemistry calculations. Despite this, and especially for simulations involving more intensive calculations, especially if the dynamics are performed for multiple electroni- 
cally excited states, a significant speed-up can be achieved and will extend the applicability of DD-vMCG simulations in terms of the size of systems understudy and the accuracy of the quantum chemistry methods which can be applied.

\section{Acknowledgements}

The author thanks the Royal Society for the Research Grant (RG150523).

\section{References}

1 G. Worth and L. Cederbaum, Annual Review Of Physical Chemistry, 2004, 55, 127-158.

2 M. H. Beck, A. Jäckle, G. A. Worth and H.-D. Meyer, Phys. Rep., 2000, 324, 1-105.

3 B. Poirier, Journal of Theoretical and Computational Chemistry, 2003, 2, 65-72.

4 S. Habershon, D. E. Manolopoulos, T. E. Markland and T. F. Miller III, Annual review of physical chemistry, 2013, 64, 387413.

5 J. O. Richardson, The Journal of chemical physics, 2015, 143, 134116.

6 J. O. Richardson, P. Meyer, M.-O. Pleinert and M. Thoss, Chemical Physics, 2016.

7 J. C. Tully and R. K. Preston, The Journal of Chemical Physics, 1971, 55, 562-572.

8 J. C. Tully, Journal of Chemical Physics, 1990, 93, 1061-1071.

9 E. J. Heller, The Journal of Chemical Physics, 1975, 62, 15441555.

10 E. J. Heller, The Journal of Chemical Physics, 1981, 75, 29232931.

11 T. J. Martinez, M. Ben-Nun and R. Levine, The Journal of Physical Chemistry, 1996, 100, 7884-7895.

12 M. Ben-Nun and T. J. Martınez, The Journal of chemical physics, 1998, 108, 7244-7257.

13 D. V. Shalashilin and M. S. Child, Chemical physics, 2004, 304, 103-120.

14 D. V. Shalashilin, The Journal of chemical physics, 2009, 130, 244101.

15 D. V. Makhov, W. J. Glover, T. J. Martinez and D. V. Shalashilin, The Journal of chemical physics, 2014, 141, 054110.

16 J. E. Stone, D. J. Hardy, I. S. Ufimtsev and K. Schulten, Journal of Molecular Graphics and Modelling, 2010, 29, 116 - 125.

17 R. M. Farber, Journal of Molecular Graphics and Modelling, 2011, 30, $82-89$.

18 A. W. Gotz, M. J. Williamson, D. Xu, D. Poole, S. Le Grand and R. C. Walker, Journal of chemical theory and computation, 2012, 8, 1542-1555.

19 I. S. Ufimtsev and T. J. Martinez, Computing in Science \& Engineering, 2008, 10, 26-34.

20 I. S. Ufimtsev and T. J. Martinez, Journal of Chemical Theory and Computation, 2008, 4, 222-231.

21 I. S. Ufimtsev and T. J. Martinez, Journal of Chemical Theory and Computation, 2009, 5, 2619-2628.

22 I. S. Ufimtsev and T. J. Martínez, Journal Of Chemical Theory
And Computation, 2008, 4, 222-231.

23 A. V. Titov, I. S. Ufimtsev, N. Luehr and T. J. Martinez, Journal of chemical theory and computation, 2012, 9, 213-221.

24 High dimensional quantum dynamics: Basic Theory, Extensions, and Applications of the MCTDH method, ed. H.-D. Meyer, F. Gatti and G. A. Worth, VCH, Weinheim, Germany, 2008.

25 M. J. Frisch, G. W. Trucks, H. B. Schlegel, G. E. Scuseria, M. A. Robb, J. R. Cheeseman, G. Scalmani, V. Barone, B. Mennucci, G. A. Petersson, H. Nakatsuji, M. Caricato, X. Li, H. P. Hratchian, A. F. Izmaylov, J. Bloino, G. Zheng, J. L. Sonnenberg, M. Hada, M. Ehara, K. Toyota, R. Fukuda, J. Hasegawa, M. Ishida, T. Nakajima, Y. Honda, O. Kitao, H. Nakai, T. Vreven, J. A. Montgomery, Jr., J. E. Peralta, F. Ogliaro, M. Bearpark, J. J. Heyd, E. Brothers, K. N. Kudin, V. N. Staroverov, R. Kobayashi, J. Normand, K. Raghavachari, A. Rendell, J. C. Burant, S. S. Iyengar, J. Tomasi, M. Cossi, N. Rega, J. M. Millam, M. Klene, J. E. Knox, J. B. Cross, V. Bakken, C. Adamo, J. Jaramillo, R. Gomperts, R. E. Stratmann, O. Yazyev, A. J. Austin, R. Cammi, C. Pomelli, J. W. Ochterski, R. L. Martin, K. Morokuma, V. G. Zakrzewski, G. A. Voth, P. Salvador, J. J. Dannenberg, S. Dapprich, A. D. Daniels, Ö. Farkas, J. B. Foresman, J. V. Ortiz, J. Cioslowski and D. J. Fox, Gaussian 09 Revision A.1, Gaussian Inc. Wallingford CT 2009.

26 J. P. Perdew, K. Burke and M. Ernzerhof, Phys. Rev. Lett., 1996, 77, 3865-3868.

27 C. Adamo and V. Barone, The Journal of chemical physics, 1999, 110, 6158-6170.

28 T. J. Frankcombe, The Journal of chemical physics, 2014, 140, 114108.

29 G. W. Richings, I. Polyak, K. E. Spinlove, G. Worth, I. Burghardt and B. Lasorne, International Reviews In Physical Chemistry, 2015, 34, 269-308.

30 A. N. Tikhonov, Dokl. Akad. Nauk SSSR, 1943, pp. 195-198.

31 I. Polyak, C. S. Allan and G. A. Worth, The Journal of chemical physics, 2015, 143, 084121.

32 I. Burghardt, H.-D. Meyer and L. Cederbaum, The Journal of chemical physics, 1999, 111, 2927-2939.

33 G. Worth, M. Robb and I. Burghardt, Faraday Discussions, 2004, 127, 307-323.

34 I. Burghardt, K. Giri and G. Worth, The Journal of chemical physics, 2008, 129, 174104.

35 M. Vacher, M. J. Bearpark and M. A. Robb, Theoretical Chemistry Accounts, 2016, 135, 1-11.

36 P. A. Dirac, Mathematical Proceedings of the Cambridge Philosophical Society, 1930, pp. 376-385.

37 I. I. Frenkel, J. I. Frenkel, J. I. Frenkel, R. Physicist, J. I. Frenkel and R. Physicien, Wave mechanics: advanced general theory, Clarendon Press Oxford, 1934.

38 I. Tavernelli, Physical Review A, 2013, 87, 042501.

39 J. C. Mullaney, D. P. Zaleski, D. P. Tew, N. R. Walker and A. C. Legon, ChemPhysChem, 2016.

40 S. Römer, M. Ruckenbauer and I. Burghardt, The Journal of chemical physics, 2013, 138, 064106. 
41 H. Wang and M. Thoss, The Journal of Chemical Physics, 2003, 119, 1289-1299.

42 B. F. Curchod, A. Sisto and T. J. Martínez, The Journal of Physical Chemistry A, 2017.

43 J. W. Snyder Jr, B. F. Curchod and T. J. Martínez, The journal of physical chemistry letters, 2016, 7, 2444-2449.

44 M. Ben-Nun, J. Quenneville and T. J. Martínez, The Journal of Physical Chemistry A, 2000, 104, 5161-5175.

45 A. B. McCoy, R. B. Gerber and M. A. Ratner, The Journal of chemical physics, 1994, 101, 1975-1987.

46 M. Braun, H. Metiu and V. Engel, The Journal of chemical physics, 1998, 108, 8983-8988.

47 T. J. Frankcombe, M. A. Collins and G. A. Worth, Chemical Physics Letters, 2010, 489, 242-247.

48 G. W. Richings and S. Habershon, Chemical Physics Letters, http://dx.doi.org/10.1016/j.cplett.2017.01.063, 2017.

49 G. Capano, T. J. Penfold, M. Chergui and I. Tavernelli, Phys. Chem. Chem. Phys., DOI: 10.1039/C7CP00436B (2017). 